\title{
The population of Magellanic Cloud planetary nebulae
}

\author{
Letizia Stanghellini \\ National Optical Astronomy Observatory, 950 N. Cherry Ave, Tucson AZ 85719, USA
}

\begin{abstract}
In this review we address the progress that has been made toward the understanding of Magellanic Cloud planetary nebulae (PNe) and their evolution since the last Magellanic Cloud Symposium. Planetary nebulae in the Magellanic Clouds are the key probes of stellar and circumstellar evolution, both for their known distances and relative vicinity, and for their broad metallicity range A selection of recent results is presented, including the HST study of $\mathrm{PNe}$ and their central stars, the study of the population of Magellanic Cloud PNe based on abundance analysis, the recent Spitzer analysis of their dust contents, and the use of Magellanic Cloud PNe to constrain the distance scale of Galactic PNe.
\end{abstract}

Keywords. stars: AGB and post-AGB, stars: evolution, stars: winds, outflows, planetary nebulae: general, Magellanic Clouds

\section{Introduction}

Planetary nebulae $(\mathrm{PNe})$ are direct probes of the evolution of low- and intermediatemass stars (LIMS, $\sim 1-8 \mathrm{M}_{\odot}$ ). LIMS constitute a major component of stellar mass in all types of galaxies and in the intra-cluster medium; they go through the asymptotic giant branch (AGB) phase, which is characterized by very high IR luminosities, high mass-loss rates, and the production of carbon and nitrogen. Through PNe, this important phase of stellar evolution is made observable across different galaxy types, and as far as $\sim 30 \mathrm{Mpc}$. The progeny of LIMS in galaxies are especially important since LIMS are the major producers of nitrogen in the universe, and they supply as much carbon as massive stars. The knowledge of AGB and PN evolution in different environments, and, in particular, at different metallicities, is essential to soundly constraint the models of stellar and Galactic evolution.

Planetary nebulae in the Magellanic Clouds have always been of great interests for the metallicity baseline that the Clouds offer, $Z \sim 0.2-1 \mathrm{Z}_{\odot}$ (Russell \& Bessell 1989). The population of Magellanic Cloud PNe is thus the benchmark for AGB studies at moderately low metallicity, essential for the understanding of the integrated light in unresolved galaxies (Maraston 2005). While the Magellanic Cloud PNe are typically 50 times farther away than their Galactic counterparts, their distance uncertainties are very low, $\sim 5 \%$ compared to $\sim 50 \%$ or more for Galactic PNe (Stanghellini et al. 2008), making the former the best absolute probes of LIMS evolution. Nonetheless, the Magellanic Cloud $\mathrm{PNe}$ are close enough to be studied in much detail, both spectroscopically and via imaging. Furthermore the low selective reddening toward the Clouds represent a further advantage with respect to the Galactic PN population.

This paper is an overview of recent results in the field of Magellanic Cloud PNe, with particular regard to space-based observations. In $\S 2$ we explore the advances in this field since last Magellanic Cloud Symposium. In $\S 3$ we discuss PN morphology in the Clouds, and how this physical property of PNe is correlated to stellar evolution. Section 4 deals 
with PN abundances and the study of their progenitor environment through the $\alpha$ elements, i.e., those elements whose yields do not change during LIMS evolution. Section 5 looks at the central stars (CS) of Magellanic Cloud PNe, who constitute the only CS population of any significant size in the universe whose physical parameters, such as luminosity and mass, are known in absolute terms. Section 6 gives an overview on recent studies on dust in Magellanic Cloud PNe, based on Spitzer observations. Section 7 shows how Magellanic Cloud PNe can forward the field of Galactic PNe also, by framing the calibration of the Galactic PN distance scale. Finally, in $\S 8$, we present a summary and future endeavors in this field.

\section{Progress since the last IAU Symposium}

In the last decade the field of Magellanic Cloud PNe has advanced greatly in several directions. The use of systematic ground-based surveys has allowed the discovery and spectroscopic confirmation of many more Magellanic Cloud PNe, more than doubling their known population size. About 230 PNe were known in the LMC (Leisy et al. 1997) at the time of the last Magellanic Clouds IAU Symposium (Dopita 1999), while recently Reid \& Parker (2006) have identified $\sim 700$ PN candidates in the LMC, $\sim 300$ of which have been spectroscopically confirmed (Reid \& Parker, this volume). In the SMC, Jacoby \& De Marco (2002) analyzed the $\sim 60$ PNe that define a complete sample 6 magnitudes down the planetary nebula luminosity function (PNLF) cutoff for the central $2.8 \mathrm{deg}^{2}$ of the SMC. This makes the SMC the first galaxy where PNe are resolved, and the PNLF features can be studied and interpreted based on a magnitude-limited, complete population. Ground-based spectroscopy is essential not only to confirm a target as a bona fide PN, but also to analyze the plasma and determine the elemental abundances. In the last decade, thanks especially to the work of Leisy \& Dennefeld (2006), and Costa et al. (2000), and Idiart et al. (2007) also, a large database of Magellanic Cloud PN abundances has become available.

Space astronomy has been essential to forward the field of Magellanic Cloud PNe. The extensive use of the HST, which has the capability of resolving them spatially, allowed morphological studies of extragalactic PNe, and made their central stars directly observable. Central stars of Magellanic Cloud PNe have been studied in detail by Villaver et al. (see $§ 5$ ), and, given the known distances of Magellanic Cloud PNe, it had been possible to locate quite accurately these stars on the $H S T$ diagram for direct comparison with the stellar evolutionary tracks. Ultraviolet spectroscopy has allowed the detection of carbon emission lines in Magellanic Cloud PNe (Stanghellini et al. 2005), and the derivation of their carbon abundances.

As Spitzer has become available, the IR spectra of Magellanic Cloud PNe became observable, affording dust studies both in imaging (Hora et al. 2008) and spectroscopy (Stanghellini et al. 2007); furthermore, Spitzer spectroscopy reaches out to many atomic transitions that are elusive in the optical, allowing precise abundance calculations (Bernard-Salas et al. 2004, 2008). Another important aspect that has advanced in the field of Magellanic Cloud PNe is the publication of sets of stellar models, both synthetic (Marigo 2001, and this volume) and evolutionary (e.g., Karakas \& Lattanzio 2007), based on initial conditions that reflect those of the Magellanic Cloud populations. These models give the yields of the element of stellar evolution in relation to the few final thermal pulses, when the $\mathrm{PN}$ is ejected, thus are readily comparable with the PN observations.

The wealth of new data and models make the Magellanic Cloud PNe the ideal laboratory to study LIMS populations and evolution at various metallicities; these have been used to explore, and trying to answer, some open questions in the field on $\mathrm{PNe}$, in 
Table 1. Magellanic Cloud PN morphology.

\begin{tabular}{lrr}
\hline & LMC & SMC \\
\hline round & $29 \%$ & $35 \%$ \\
elliptical & $17 \%$ & $29 \%$ \\
bipolar & $34 \%$ & $6 \%$ \\
bipolar core & $17 \%$ & $24 \%$ \\
point-symmetric & $3 \%$ & $6 \%$ \\
\hline
\end{tabular}

particular, the focus has been on the different PN morphologies, and how these originate and evolve; the chemistry of PNe as they evolve in different metallicity environments, and how do they contribute to cosmic recycling; the evolution of the PN central stars (CS); the PNLF in the Magellanic Clouds, and how can it be used to constrain and scale the extragalactic distance scale; the role of dust in PNe in PN ejection, evolution, and morphology, and the nature of dust in PNe of different metallicities; finally the use of Magellanic Cloud PNe as calibrator of the Galactic PN distance scale. In the next few sections we will explore several of these topics.

\section{Magellanic Cloud PN morphology and the evolutionary connection}

Planetary nebula morphology reveals the evolutionary history of the final phases of the LIMS life. Magellanic Cloud PNe are on average 0.5 arcsec across, thus they are spatially resolvable only with observations from space. In the last decade many samples of Magellanic Cloud PN images have been acquired with the HST, forming a large database to study the morphological evolutionary connection (Shaw et al. 2001; Stanghellini et al. 2003; Shaw et al. 2006). These studies include 114 LMC and 35 SMC PNe, representing approximately $2 / 3$ of all Magellanic Cloud PNe known at the time of these surveys, and populate the 5 bright magnitude bins of the PNLF (in terms of $m_{\lambda 5007}$ ). Shapes of Magellanic Cloud PNe can be grouped into four major classes, round, elliptical, bipolar, and bipolar core (or ring) PNe, just as their Galactic counterparts. In Table 1 we show the statistics of the morphological types of Magellanic Cloud PNe, where we enlarge the sample described above by including the PNe already observed with the HST by Dopita, Vassiliadis, and collaborators (see Dopita 1999) We note that asymmetric PNe (bipolar $\mathrm{PNe}$, and those ones showing other asymmetries such as bipolar cores) are much more common in the LMC than the SMC, and, in particular, the number of bipolar PNe in the SMC is very low.

The origin of PN morphology can be ascribed to the mechanism of mass ejection at the tip of the AGB and to the condition of the circumstellar medium at that evolutionary phase: The round and most elliptical shapes can be formed, according to hydrodynamic models, via ballistic expansion. On the other hand, the evolution into bipolar shape of the AGB ejecta needs the presence of an equatorial enhancement, at the time of the envelope ejection. What creates the conditions for the enhancement is still controversial. Most of the models for highly asymmetric PN evolution involve either rotation and magnetic fields (García-Segura 1997), or common-envelope (CE) processes (Morris 1981; Soker 1998). The former set of models agree with a progenitor-mass dependency of morphological evolution, while the latter set of models, those involving the CE evolution, are mass independent, since the chance of close binary evolution unlikely depends on the progenitor mass.

The Magellanic Cloud data have helped to clarify that the process forming bipolar $\mathrm{PNe}$ is necessarily mass dependent for the majority of observed bipolar PNe. It had been shown that asymmetry is related to higher LIMS mass. From stellar evolution 
we know that the third dredge-up, and the hot bottom burning process, occur only in the most massive AGB stars $\left(\mathrm{M} / \mathrm{M}_{\odot}>3-4\right)$. These processes have the net effect to reduce carbon and enhance nitrogen (and N/O) both in the Magellanic Cloud and the solar-metallicity models (Marigo 2001; Karakas \& Lattanzio 2007). In Table 2 we show the average abundances of the key evolutionary elements for those Magellanic Cloud PNe whose morphology have been classified via the HST images. The abundances are from Leisy \& Dennefeld (2006) excluding uncertain values. Carbon abundances are also from Stanghellini et al. (2005) and from new ACS prism spectra (Stanghellini et al., in preparation). The averages are given for whole samples, and for the morphological groups of symmetric (round and elliptical), and asymmetric (bipolar core and bipolar), PNe. For the LMC, the sample of bipolar PNe is large enough to have a separate entry for this specific type. It is evident that PN chemistry closely correlates to their morphology. A look at the LMC averages in Table 2 shows that carbon is depleted in asymmetric PNe, whereas nitrogen is strongly enriched.

Figure 1 shows the averages and ranges of the $\mathrm{N} / \mathrm{H}$ ratios in $\mathrm{SMC}(Z=0.004)$, LMC $(Z=0.008)$, and Galactic $(Z=0.016) \mathrm{PNe}$, plotted against the galaxy metallicity. The Galactic data are from Stanghellini et al. (2006). There is no doubt that, whatever makes the asymmetric PNe acquire their shape, it has to be closely correlated with the nitrogen yield, and this, in turn, is correlated with the progenitor mass, for all metallicities. There are few bipolar PNe with low nitrogen (and/or high carbon) abundance. In these cases, the mechanism forming the bipolarity is mass-independent, and could be ascribed to CE evolution, following close binary interaction. Interestingly, binary evolutionary models show that close binary evolution does not enhance nitrogen nor deplete carbon (Izzard

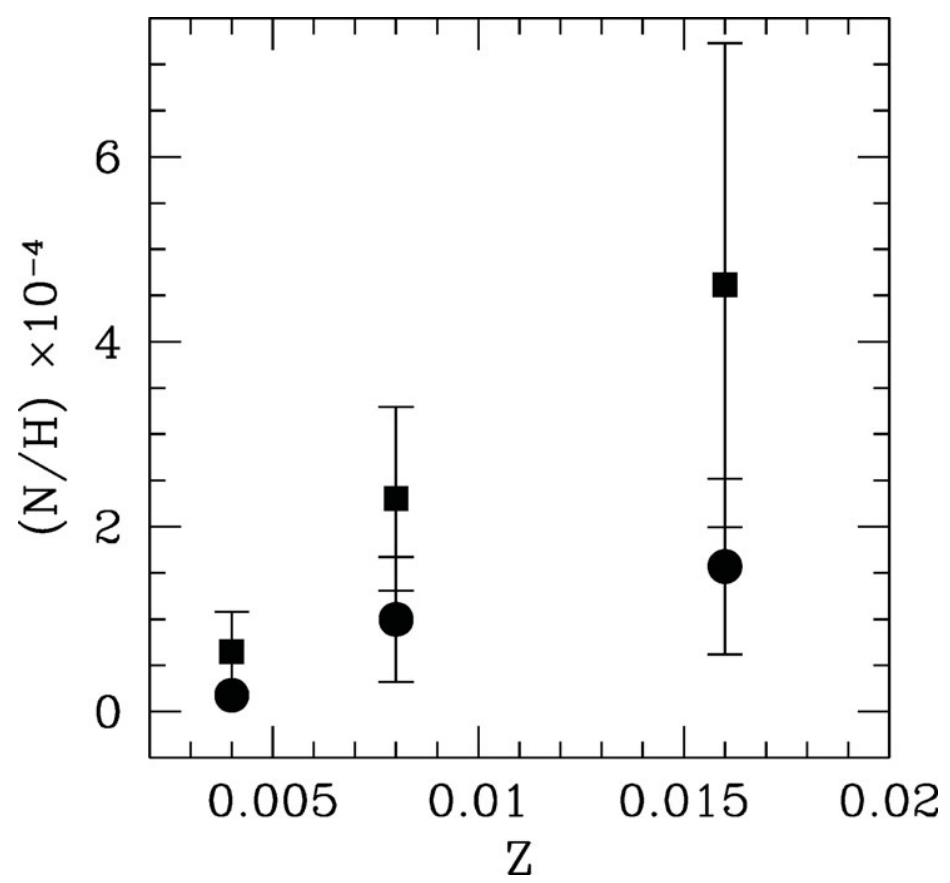

Figure 1. Average nitrogen abundances in the populations of SMC, LMC, and Galactic PNe with known morphology, plotted against the mean galaxy metallicity. Averages are plotted with filled circles and squares for symmetric (round and elliptical) and asymmetric (bipolar core and bipolar) PNe respectively. Bars represent data ranges. 
Table 2. Evolutionary connection.

\begin{tabular}{lrr}
\hline & LMC & SMC \\
\hline$<\mathrm{C} / \mathrm{H}>\times 10^{4}$ & & \\
whole sample & $2.49 \pm 2.18$ & $3.71 \pm 3.66$ \\
round, elliptical & $3.96 \pm 2.00$ & $4.55 \pm 3.86$ \\
bipolar core, bipolar & $2.10 \pm 2.13$ & 2.00 \\
bipolar & $0.47 \pm 0.45$ & $\ldots$ \\
& & \\
$<\mathrm{N} / \mathrm{H}>\times 10^{4}$ & & \\
whole sample & $1.48 \pm 1.75$ & $0.29 \pm 0.33$ \\
round, elliptical & $1.00 \pm 1.35$ & $0.18 \pm 0.68$ \\
bipolar core, bipolar & $2.30 \pm 1.99$ & $0.65 \pm 0.87$ \\
bipolar & $2.68 \pm 2.11$ & $\cdots$ \\
& & \\
$<\mathrm{N} / \mathrm{O}>$ & & \\
round, elliptical & $0.57 \pm 0.89$ & $0.12 \pm 0.09$ \\
bipolar core, bipolar & $1.31 \pm 1.45$ & $\ldots$ \\
bipolar & $1.54 \pm 1.61$ & $\cdots$ \\
\hline
\end{tabular}

et al. 2006), thus can not describe the formation of the majority of SMC, LMC, and Galactic bipolar PNe.

\section{Abundances in Magellanic Cloud PNe, and metallicity gradients}

The abundance of oxygen, neon, and other $\alpha$-elements in Magellanic Cloud PNe probes the chemical environment at the time of PN progenitor formation, since $\alpha$-elements can be considered primordial, as they are mostly produced in massive stars in primary nucleosynthesis, and not in LIMS. From the literature selection described in the previous section we looked at the primordial element distribution in Magellanic Cloud PNe. In Figure 2 we show the distribution of oxygen and neon in the LMC (open symbols) and the SMC (filled symbols) PNe, where morphological types have been also coded. The correlation is well defined, as expected from elements in lockstep evolution. The average $\mathrm{Ne} / \mathrm{O}$ ratio is $\langle\mathrm{Ne} / \mathrm{O}\rangle=0.17 \pm 0.09$ both for the $\mathrm{LMC}$ and the SMC, while it is 0.27 in Galactic PNe (Stanghellini et al. 2006). The lower ratio at low metallicity shows that oxygen and neon abundances do not always scale with metallicity in lockstep. We have checked the PN abundance distribution of $\alpha$-elements across the face of the Clouds, looking for a metallicity gradient. We have not found a clear metallicity gradient in either Cloud, nor a relation between $\alpha$-element abundance and the location of the star forming regions. The morphological type distribution across the LMC has also been studied (Stanghellini et al. 2002) to show no particular morphological segregation, in agreement with a short crossing time of these galaxies compared to the timeframe of LIMS evolution.

\section{Central stars of Magellanic Cloud PNe}

The direct imaging of central stars (CS) of Magellanic Cloud PNe is only possible through space imaging. Villaver et al. (2003, 2004, 2007) have analyzed 50 LMC and SMC CS images acquired with STIS and WFC2 on the HST, and, by measuring their luminosity and temperature (with the aid of ground-based spectroscopy), estimated the 
masses of $\sim 20$ CS. Villaver et al. (2007) determined that $\left\langle\mathrm{M} / \mathrm{M}_{\odot}>=0.65 \pm 0.1\right.$ both for the LMC and the SMC PNe, which is slightly higher than what has been estimated for Galactic CS. The possible reason why Magellanic Cloud CS are more massive than their Galactic counterparts is that, at lower metallicity, the mass loss at the TP-AGB is less efficient (Villaver et al. 2003, 2004). Interestingly, Villaver et al. found no clear cut relation between stellar mass and PN morphology, indicating that by the time the superwind is over there is no longer memory of the initial stellar mass.

The CS of the Magellanic Cloud PNe are among the few CS with known distances. As such, they offer the opportunity to test the PNLF, and, in particular, to see which type of stars illuminates the PNe that populate the high luminosity cutoff of the PNLF, which is used to calibrate the extragalactic distance scale. In Figure 3 we show the relation between the measured PN radii and the CS luminosities in the Clouds. Stellar and nebular data are from the papers quoted above. We see that the brightest CS are those hosted by compact PNe in both the LMC and the SMC, with $R_{\text {phot }}<0.5$ pc. This is expected, as the evolution of central stars (on the HR diagram) follows a luminosity plateau right after PN ejection, and then evolves toward the WD cooling line. Nonetheless, this effect has never been shown empirically before, since the distance of Galactic PNe are too uncertain for these types of studies. Central stars have masses between $\sim 0.55$ and 1.4 $\mathrm{M}_{\odot}$. We found that the stars populating the bright end of the PNLF are those with $M \sim 0.65-0.7 \mathrm{M}_{\odot}$, rather than the most massive ones.

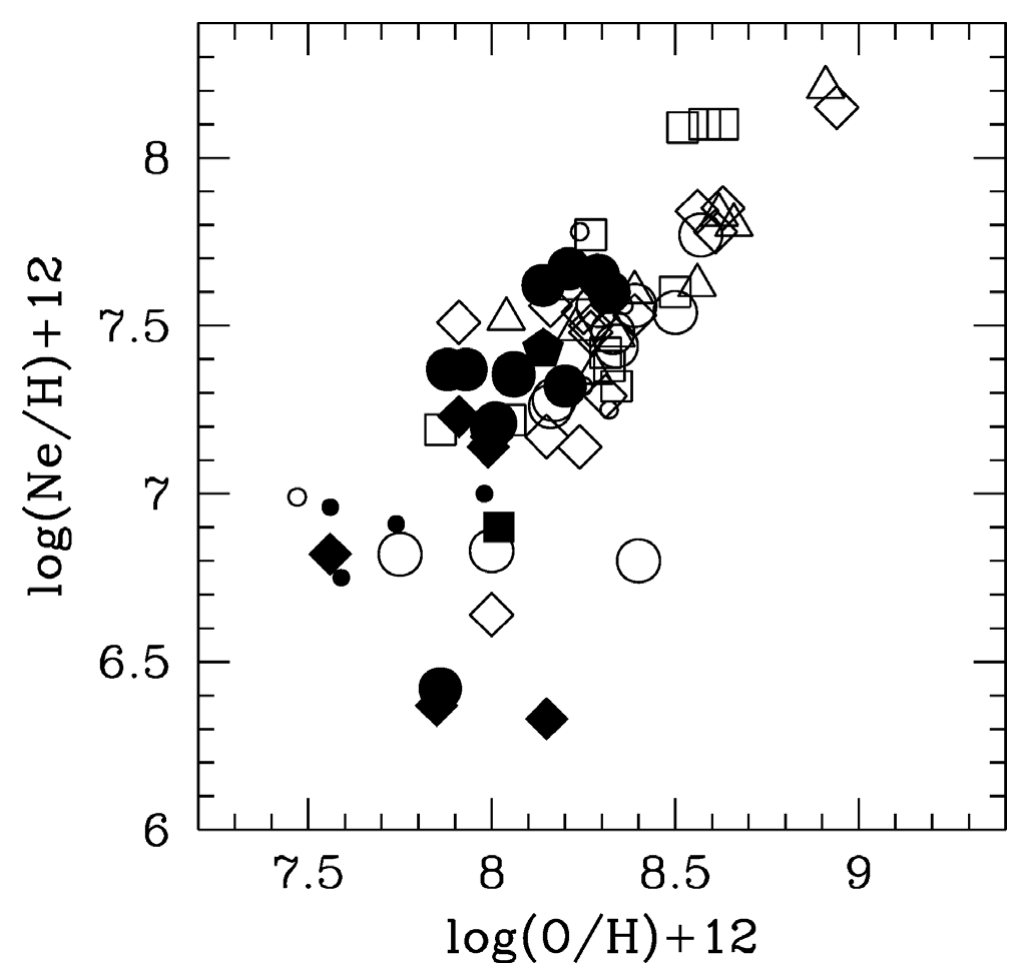

Figure 2. Neon vs. oxygen abundances, in log scale, for LMC (open symbols) and SMC (filled symbols) PNe. Shape of symbol indicates morphological type; Circle: round, diamonds: elliptical, triangles: bipolar core, squares: bipolar, and small circles: unknown morphology PNe. 


\section{Dust in Magellanic Cloud PNe}

The study of dust in Magellanic Cloud PNe had become possible with the launch of the Spitzer Space Telescope; only a few bright Magellanic Cloud PNe were within observing reach with earlier technology. The IRS/Spitzer spectra in the 1-4 $\mu \mathrm{m}$ range had proven essential to study both the circumstellar dust and gas (Bernard-Salas et al. 2004, 2008; Stanghellini et al. 2007) at a distance of $\sim 50 \mathrm{kpc}$. The importance of studying dust in $\mathrm{PNe}$ resides in the fact that mass loss occurring toward the end of the AGB phase is still not completely understood. Theoretical studies indicate that the pressure on the dust grains produces the mass loss at the AGB tip (Willson 2000), and that mass-loss efficiency is directly proportional to metallicity, through the dependence of the absorption coefficient. The data available to date seem to agree with this correlation: there are fewer obscured AGB stars in the Magellanic Clouds than in the Galaxy (Groenewegen et al. 2000); the C-rich to O-rich ratio of AGB stars is higher at lower metallicity (Cioni \& Habing 2003); and, as discussed in $\S 3$, there are fewer aspheric PNe in the SMC than in the LMC, showing that asymmetry is rarer in lower metallicity environments.

On this basis, Stanghellini et al. (2007) examined a homogeneous sample of IRS spectra to determine the IR/dust properties of the Magellanic Cloud PNe whose morphology had been previously determined through the HST images. Half of the analyzed spectra are featureless, except for the nebular emission lines and a weak dust continuum; the other half shows dust features in the form of solid state emission superimposed on the dust continuum. In most cases the solid state features are recognized as carbon-rich dust emission such as $\mathrm{SiC}$ and $\mathrm{PAH}$, while oxygen-rich dust signatures were observed only in three PNe.

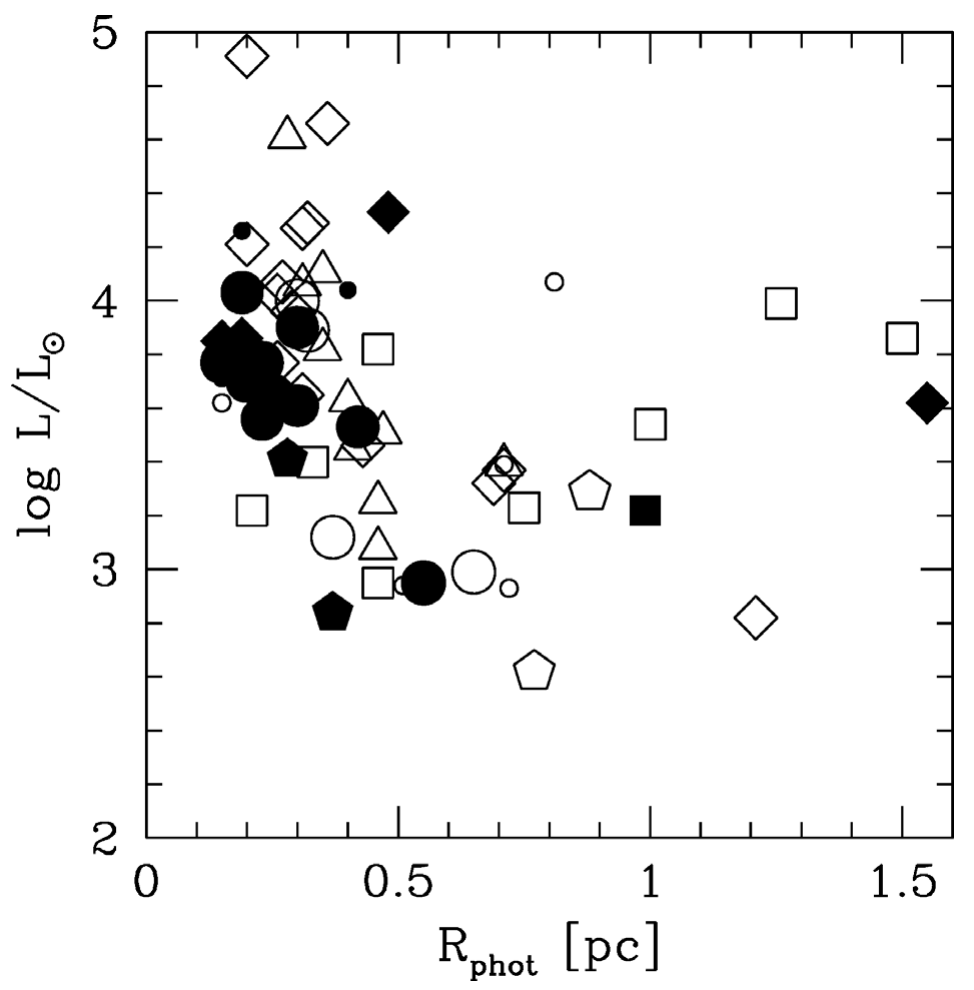

Figure 3. CS luminosities vs. PN physical radii, for LMC (open symbols) and SMC (filled symbols) PNe. Shape of symbol indicates morphological type as in Fig. 2. 
In Figure 4 we show how the dust features in the IRS spectra correlate with the chemistry of nebular gas, and with morphology. Both panels show the IR luminosity (derived from a black-body fit of the dust continua, after subtracting the solid state features and the nebular emission lines) versus the carbon abundance of the PNe. Symbols in the left panel characterize the dust properties, while on the right panel they indicate the different PN morphologies. Symbols in the right panel are the same as in Figures 2 and 3 , while in the left panel triangles indicate featureless, diamonds carbon-rich dust, and squares oxygen-rich dust PNe from the IRS spectra. In both panels, open symbols indicate LMC, and filled symbols SMC, PNe. From the left panel we infer that most featureless IRS spectra PNe (triangles) are in the lower luminosity part of the diagram, possibly indicating the rather short time span in which solid state features can be observed after $\mathrm{PN}$ ejection. This is also confirmed by the dependency of the features on the nebular radii (Stanghellini et al. 2007). It is evident from the Figure that the carbon-rich dust $\mathrm{PNe}$ (diamonds) correspond to higher carbon abundances, and oxygen-rich dust PNe (squares) are those with low carbon. The right panel shows that all round and elliptical PNe (circles and diamonds) correspond to carbon-rich dust, or featureless, IRS spectra, and none of the oxygen-rich dust $\mathrm{PNe}$ are round or elliptical. The differences in gas and morphology between LMC and SMC PNe is clear in Figure 4, where most of the SMC PNe whose carbon abundances and IRS spectra are available are either round or elliptical, with carbon-rich dust.

Comparison of the IRS data with the literature disclosed a sharp difference between dust features in the Magellanic Cloud and Galactic PN populations, in that all observed Galactic PNe show solid state features (García-Lario et al., in preparation). The ratio of carbon-rich dust to oxygen-rich dust $\mathrm{PNe}$ is $\sim 11$ in the SMC, $\sim 4.5$ in the LMC, while it is estimated to be close to unity in the Galaxy (García-Lario et al., in preparation), indicating that the population metallicity has enormous impact on AGB dust formation.

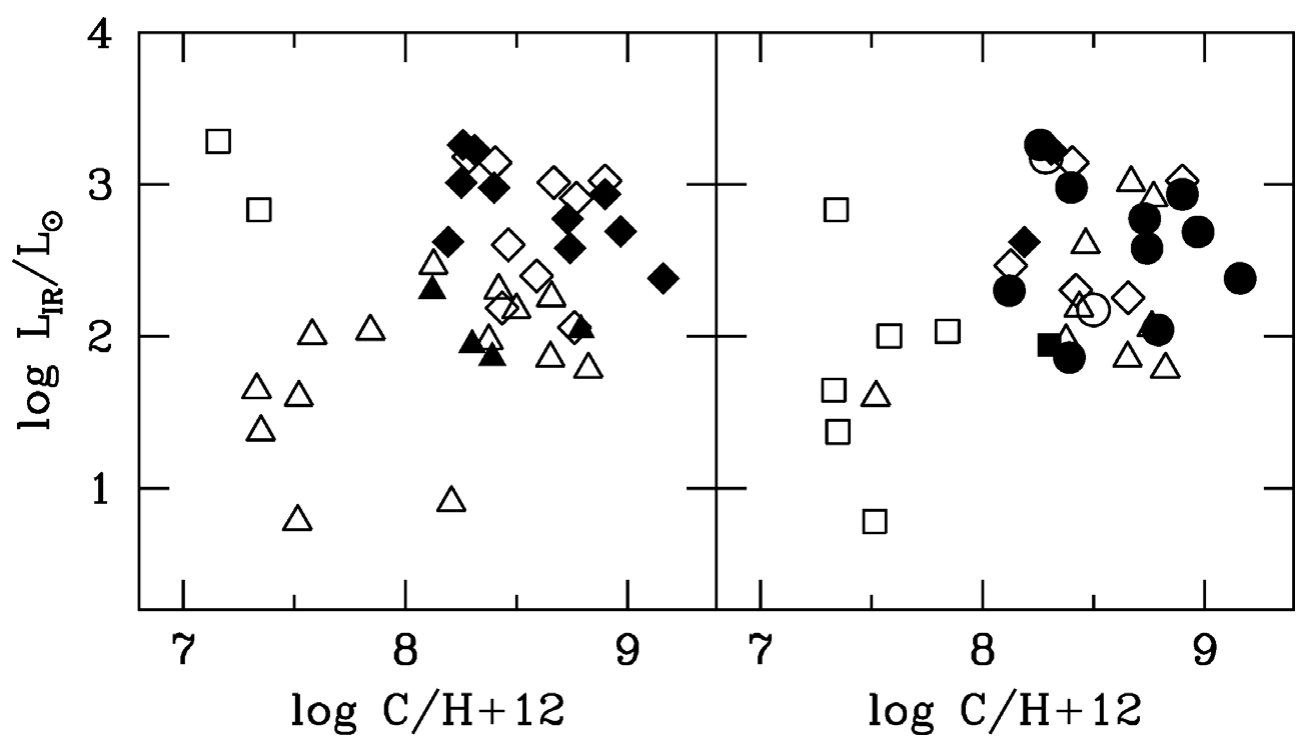

Figure 4. IR Luminosity vs. carbon abundance of Magellanic Cloud PNe. Left panel: dust properties (triangles: no dust features; diamonds: carbon-rich dust; and squares: oxygen-rich dust PNe). Right panel: morphology (circles: round; diamonds: elliptical; squares: bipolar, triangles: bipolar core PNe). In both panels, open symbols are for LMC, and filled symbols for SMC, PNe (adapted from Stanghellini et al. 2007). 


\section{Magellanic Cloud PNe as distance calibrators}

There are $\sim 1800$ PNe in the Galaxy (Acker et al. 1992), but their distances are uncertain with the exception of a few PNe where a cluster or binary membership could be established. To get the distances of most Galactic PNe it is customary to use relations between physical parameters, and calibrate these relations with the parameters of the few PNe with known distances. The most recent calibrations of the surface-brightness to ionized-mass scale (Daub 1982; Cahn et al. 1992) constitute the most used catalogs of PN distances. Nonetheless, even the best calibrations are based on very few data points, as model-independent Galactic PNe distances are few. On the other hand, there are more than a hundred PNe in the Magellanic Clouds whose ionized-mass and surface-brightness are available through $H S T$ observations, and they can be used to calibrate the Galactic scale. Stanghellini et al. (2008) have shown that the re-calibration is reliable, and found distances for hundreds of Galactic PNe with the new scale. The resulting distances are in excellent agreement with the Galactic parallaxes and cluster membership distances.

\section{Future endeavors}

The past decade has proven incredibly productive in the field of Magellanic Cloud PNe, especially thanks to space and ground-based surveys that have become available. Large databases have allowed the exploration of the PN abundances, yields, central stars, evolution and tracing of the different populations of PNe. Systematic studies of LMC and SMC PNe disclose the tight connection between dust, gas, and morphology. Dust production in AGB and post-AGB stars is directly related to metallicity. It seems that, for the most part, symmetric PNe are the progeny of the lower end of the LIMS mass range, while bipolarity is associated with the higher-mass progenitors, thus the mechanism that produces bipolarity must depend on progenitor mass, in most observed cases.

An initial set of abundances have become available in this decade, and hopefully will be expanded soon to include all known Magellanic Cloud PNe. The new abundance databases will include the IR emission lines, thus be less dependent on the ionization correction factor modeling that is typically used to obtain elemental concentrations. The use of a refurbished HST will be very important to extent the morphological approach. There are many other aspects of the Magellanic Cloud PN field that could not have been explored in this paper, but in particular the comparison between the PN properties, and those of their progenitors (AGB stars) and progeny (WD) in a complete evolutionary scheme is the most interesting avenue for future discoveries. Models of the stars and nebulae together, including dust, will eventually encompass observations of the whole life and death of the LIMS at different metallicities, to directly understand the contribution of LIMS to galaxies in terms of their mass, metallicity, and observing wavelength.

\section{Acknowledgements}

Thanks to the Organizers for a very interesting Symposium, and to my collaborators for their input in the work presented here.

\section{References}

Acker, A., Marcout, J., Ochsenbein, F., Stenholm, B., \& Tylenda, R. 1992, Strasbourg - ESO catalogue of galactic planetary nebulae (Garching: European Southern Observatory)

Bernard-Salas, J., Houck, J. R., Morris, P. W., Sloan, G. C., Pottasch, S. R., \& Barry, D. J. 2004, ApJS, 154, 271 
Bernard-Salas, J., Pottasch, S. R., Gutenkunst, S., Morris, P. W., \& Houck, J. R. 2008, ApJ, 672,274

Cahn, J. H., Kaler, J. B., \& Stanghellini, L. 1992, A\&SAS, 94, 399

Cioni, M. -R. L. \& Habing, H. J. 2003, A\&A, 402, 133

Costa, R. D. D., de Freitas Pacheco, J. A., \& Idiart, T. P. 2000, A $\& A S, 145,467$

Daub, C. T. $1982, A p J, 260,612$

Dopita, M. A. 1999, in Y. -H. Chu, N. Suntzeff, J. Hesser, \& D. Bohlender (eds.), New Views of the Magellanic Clouds, IAUS 190, p. 332

García-Segura, G. 1997, ApJ, 489, L189

Groenewegen, M. A. T., van Loon, J. T., Whitelock, P. A., Wood, P. R., \& Zijlstra, A. A. 2000, in R. F. Wing (ed.), The Carbon Star Phenomenon, IAUS 177 (Dordrecht: Kluwer), p. 385

Hora, J. L., Cohen, M., Ellis, R. G., et al. 2008, AJ, 135, 726

Idiart, T. P., Maciel, W. J., \& Costa, R. D. D. 2007, A\& A, 472, 101

Izzard, R. G., Dray, L. M., Karakas, A. I., Lugaro, M., \& Tout, C. A. 2006, A\&\&A, 460, 565

Jacoby, G. H. \& De Marco, O. 2002, AJ, 123, 269

Karakas, A. \& Lattanzio, J. C. 2007, PASA, 24, 103

Leisy, P. \& Dennefeld, M. 2006, A\&A, 456, 451

Leisy, P., Dennefeld, M., Alard, C., \& Guibert, J. 1997, A\& $A S$, 121, 407

Maraston, C. 2005, MNRAS, 362, 799

Marigo, P. 2001, $A \& A, 370,194$

Morris, M. 1981, ApJ, 249, 572

Reid, W. A. \& Parker, Q. A. 2006, MNRAS, 373, 521

Russell, S. C. \& Bessell, M. S. 1989, ApJS, 70, 865

Shaw, R. A., Stanghellini, L., Mutchler, M., Balick, B., \& Blades, J. C. 2001, ApJ, 548, 727

Shaw, R. A., Stanghellini, L., Villaver, E., \& Mutchler, M. 2006, ApJS, 167, 201

Soker, N. 1998, ApJ, 496, 833

Stanghellini, L., Shaw, R. A., Mutchler, M., Palen, S., Balick, B., \& Blades, J. C. 2002, ApJ, 575,178

Stanghellini, L., Shaw, R. A., Balick, B., Mutchler, M., Blades, J. C., \& Villaver, E. 2003, ApJ, 596,997

Stanghellini, L., Shaw, R. A., \& Gilmore, D. 2005, ApJ, 622, 294

Stanghellini, L., Guerrero, M. A., Cunha, K., Manchado, A., \& Villaver, E. 2006, ApJ, 651, 898

Stanghellini, L., García-Lario, P., García-Hernández, D. A., Perea-Calderón, J. V., Davies, J. E., Manchado, A., Villaver, E., \& Shaw, R. A. 2007, ApJ, 671, 1669

Stanghellini, L., Shaw, R. A., \& Villaver, E. 2008, ApJ, 689, 194

Villaver, E., Stanghellini, L., \& Shaw, R. A. 2003, ApJ, 597, 298

Villaver, E., Stanghellini, L., \& Shaw, R. A. 2004, ApJ, 614, 716

Villaver, E., Stanghellini, L., \& Shaw, R. A. 2007, ApJ, 656, 831

Willson, L. A. 2000, ARAA, 38, 573 